\title{
Using Social Marketing as a Tool to Increase Helmet Use among Bicycle-share Riders in Urban Settings \\ Danna Ethan ${ }^{1}$ and Corey H Basch ${ }^{2 *}$
}

${ }^{1}$ Department of Health Sciences, Lehman College, The City University of New York, New York, New York, USA

${ }^{2}$ Department of Public Health, William Paterson University, Wayne, New Jersey, USA

In urban settings, bicyclists must often contend with heavy traffic, other riders, distracted pedestrians, and other variables that increase the risk of injury and death. In 2012, for example, bicycle riders in New York City sustained over 3,800 injuries and 18 deaths due to motor vehicles alone [1]. Despite these risks, urban bicycle riding has become increasingly popular, particularly within public bicycle-share programs, which have been instituted in several cities in recent years.

The use of helmets has been shown to be effective in preventing head injuries sustained from bicycle riding accidents [2]. Despite the benefits of this health behavior, helmet use among riders in bicyclesharing programs has been shown to be remarkably low across a number of U.S. cities. In a 2013 study, over $85 \%$ of the 1,054 New York City bicycle-share riders were observed not wearing helmets regardless of the location, time of day or day of the week [3]. Similar findings from a 2012 study were reported in Boston and Washington, DC with over $80 \%$ of cyclists in these programs riding without helmets [4]. Public health literature offers little about perceived social, personal and logistical barriers to helmet use among bicycle-share riders. Studies of helmet use in other bicycle users in the United States and Netherlands indicate numerous factors that affect adult riders' likelihood of wearing helmets including physical discomfort when wearing a helmet, nonhelmet use among peers, and riders not owning a helmet $[5,6]$. Other noted perceived barriers included having an unfavorable appearance while wearing a helmet and finding it unnecessary to wear one $[5,6]$.

Educational and policy-related approaches have been implemented to increase helmet use among riders in urban bicycle-sharing programs in the United States (although no published research on the efficacy of these interventions exists). Recommendations for wearing a helmet have been incorporated into contract and promotional language, and distribution of coupons, giveaway events, and helmet rentals are also aimed at encouraging increased use $[7,8]$. In concert with efforts like these social marketing campaigns implemented by urban health and city government agencies may be a helpful tool in communicating health messages to bicycle-share participants.

A health communication approach that applies marketing practices to influencing attitudes and behaviors of a target population, social marketing has been used to address population-based health problems such as teen pregnancy, substance abuse, diabetes and obesity [9]. Program planning and evaluation are used to identify and assess intended outcomes, which can include changes in attitude, perception, knowledge and behavior. Similar to commercial marketing, this approach includes 1) analysis and segmentation of the target population, 2) using behavior change theories to guide planning, 3) tailoring health messages through audience feedback, 4) selecting appropriate communication channels, and 5) using evaluation tools to measure program effectiveness [10]. Building "brand equity" can also be used within this framework by influencing the target population to associate the campaign and its specific attributes with healthy behaviors [11]. As noted by Evans et al. [11], this tactic has been successfully implemented by the American Legacy Foundation's truth ${ }^{\circledR}$ campaign which has been associated with changes in teens' attitudes, beliefs and behaviors associated with tobacco use $[11,12]$.

Given the documented barriers to helmet use that are related to riders' perceptions (e.g. unfavorable appearance, no need to wear one, etc.), building brand equity may be a possible key strategy in changing perceptions of helmet use in urban bicycle-share programs. In this case, helmet use is the "product" to be sold by marketing not only as a practical means of reducing head injury but as a health-enhancing lifestyle choice for frequent riders. Targeted health messaging can include audience-tested appealing images of helmeted riders. Additional health communication efforts can incorporate social media to help disseminate the health message (or promote the product), include partnerships with bicycle helmet companies with a focus on helmet style, and recruit riders to serve as innovators who actively use and promote this "product." This multimodal approach communicates the message to riders that wearing a helmet is the preferred choice.

Effective social marketing campaigns involve extensive program planning that includes audience research, development, testing, and launching of the "product" as well as process and impact evaluation to determine changes in knowledge, attitudes, skills, and/or behaviors [13]. As an example, a small study of university students that analyzed the effects of a social marketing campaign found a positive change in helmet use [14]. Student focus groups generated the logo and slogan ("The Grateful Head") for the program's helmets. In addition, helmetusing student bicyclists were trained as "peer agents" who offered relevant information, encouraged fellow students to sign a helmet-use pledge card, and distributed free helmet coupons to those who gave their pledge. Mean helmet use almost doubled (27.6\% to $49.3 \%)$ by the end of the intervention.

Among the population of urban bicycle-share riders, it is essential to assess the various factors that prevent and promote this behavior before the low rate of helmet use can be adequately addressed. In addition, various subgroups of riders must be identified (e.g. commuters, tourists, weekend users) in order to target specific needs (one-time versus frequent helmet use) [15]. To address these needs, a social marketing campaign for urban bicycle-share riders may serve as a meaningful component of a comprehensive health promotion program that aims to remove barriers of and educate riders about helmet use, and to promote a lifestyle option that "sells" helmet use as a product worth investing in.

${ }^{*}$ Corresponding author: Corey $\mathrm{H}$ Basch, Department of Public Health, William Paterson University, USA, Tel: 973-720-2603; E-mail: BASCHC@wpunj.edu

Received November 20, 2013; Accepted November 21, 2013; Published November 22, 2013

Citation: Ethan D, Basch CH (2013) Using Social Marketing as a Tool to Increase Helmet Use among Bicycle-share Riders in Urban Settings. J Mass Communicat Journalism 3: e147. doi:10.4172/2165-7912.1000e147

Copyright: (C) 2013 Ethan D, et al. This is an open-access article distributed under the terms of the Creative Commons Attribution License, which permits unrestricted use, distribution, and reproduction in any medium, provided the original author and source are credited. 
Citation: Ethan D, Basch CH (2013) Using Social Marketing as a Tool to Increase Helmet Use among Bicycle-share Riders in Urban Settings. J Mass Communicat Journalism 3: e147. doi:10.4172/2165-7912.1000e147

\section{References}

1. Bicycle Crash Data 2012.

2. Thompson DC, Rivara F, Thompson R (2009) Helmets for preventing head and facial injuries in bicyclists. Cochrane Database of Systematic Reviews Issue 4: CD001855.

3. Basch CH, Ethan D, Rajan S, Samayoa-Kozlowsky S, Basch CE (2013) Helmet use among riders using the Citi Bike bicycle-sharing program: A pilot study in New York City. Journal of Community Health Published online 01 November 2013.

4. Fischer CM, Sanchez CE, Pittman M, Milzman D, Volz KA, et al. (2012) Prevalence of bicycle helmet use by users of public bikeshare programs. Annals of Emergency Medicine 60: 228-231.

5. Villamor E, Hammer S, Martinez-Olaizola A (2008) Barriers to bicycle helme use among Dutch paediatricians. Child: Care, Health and Development 34: 743-747.
6. Finnoff JT, Laskowski ER, Altman KL, Diehl NN (2001) Barriers to bicycle helmet use. Pediatrics 108: E4.

7. McKernan B (2013) No helmets required for bike share in busy NYC.

8. Helmet Rentals.

9. Centers for Disease Control. Social Marketing Resources.

10. Evans WD (2006) How social marketing works in health care. BMJ 332: 12071210.

11. Evans WD, Price S, Blahut S (2005) Evaluating the truth ${ }^{\circ}$ brand. Journal of Health Communication 10: 181-192.

12. Legacy. Keeping young people from using tobacco.

13. Centers for Disease Control. Gateway to Health Communication \& Social Marketing Practice.

14. Ludwig TD, Buchholz C, Clarke SW (2005) Using social marketing to increase the use of helmets among bicyclists. The Journal of American College Health 54: $51-58$ 